\title{
Continuous Control Set Nonlinear Model Predictive Control of Reluctance Synchronous Machines
}

\author{
Andrea Zanelli, Julian Kullick, Hisham Eldeeb, Gianluca Frison, Christoph Hackl, Moritz Diehl
}

\begin{abstract}
In this paper we describe the design and implementation of a current controller for a reluctance synchronous machine based on continuous set nonlinear model predictive control. A computationally efficient grey box model of the flux linkage map is employed in a tracking formulation which is implemented using the high-performance framework for nonlinear model predictive control acados. The resulting controller is validated in simulation and deployed on a $\mathrm{CSPACE}$ realtime system connected to a physical reluctance synchronous machine. Experimental results are presented where the proposed implementation can reach sampling times in the range typical for electrical drives and can achieve large improvements in terms of control performance with respect to state-of-the-art classical control strategies.
\end{abstract}

Index Terms-predictive control, electric motors, nonlinear systems.

\section{INTRODUCTION}

$\mathbf{I}$ $\mathrm{N}$ recent years, reluctance synchronous machines (RSMs) have emerged as a competitive alternative to classical synchronous machines (SMs) with permanent magnet (PMSMs) or direct current excitation. In addition to the favourable properties of SMs in general, e.g., high efficiency, reliability and compact design, RSMs are often easier to manufacture and comparably cheap due to the absence of magnets. Moreover, their anisotropic magnetic path in the rotor, makes them particularly suitable for saliency-based encoderless control [35, 36]. However, a major drawback of the RSM concerning control is its characteristic nonlinearity of the flux linkage, caused by magnetic saturation and cross-coupling effects in the rotor. As a consequence, the machines' inductances vary significantly with the stator currents. Moreover, additional coupling

This research was supported by the German Federal Ministry for Economic Affairs and Energy (BMWi) via eco4wind (0324125B) and DyConPV (0324166B), by DFG via Research Unit FOR 2401 and by the EU via ITNAWESCO (642 682)

Andrea Zanelli is with the Systems Control and Optimization Laboratory, Department of Microsystems Engineering, University of Freiburg, Freiburg, Germany (email: andrea.zanelli@imtek.de).

Julian Kullick is with the Department of Electrical Engineering and Information Technology at the Munich University of Applied Sciences, Munich, Germany (email: julian.kullick@hm.edu).

Hisham Eldeeb is with IAV GmbH, Munich, Germany (email: hisham.eldeeb@iav.de).

Gianluca Frison is with the Systems Control and Optimization Laboratory, Department of Microsystems Engineering, University of Freiburg, Freiburg, Germany (email: gianluca.frison@imtek.de).

Christoph M. Hackl is with the Department of Electrical Engineering and Information Technology at the Munich University of Applied Sciences, Munich, Germany (email: christoph.hackl@hm.edu).

Moritz Diehl is with the Systems Control and Optimization Laboratory, Department of Microsystems Engineering and Department of Mathematics, University of Freiburg, Freiburg, Germany (email: moritz.diehl@imtek.unifreiburg.de) between the stator $\mathrm{d}$ - and q-currents is imposed by the cross-coupling inductances and the coupling of the nonlinear back electro-motive force in the synchronous reference frame, which generally requires further measurements to be carried out online.

Regarding the control of RSMs, two main concepts have been pursued in the past: (i) Direct Torque Control (DTC) [4, 34] and (ii) field-oriented control (FOC) [3, 37, 53]. While DTC is known for its robustness and fast dynamics [5], it produces a high current distortion leading to torque ripples [6]. In contrast, vector control improves the torque response [48] and the efficiency of the system [32], but good knowledge of the system parameters is required for implementation. In [27], a completely parameter-free adaptive PI controller is proposed which guarantees tracking with prescribed transient accuracy. The controller is applied to current control of (reluctance) synchronous machines, but measurement results are not provided. In [48] and [54], the inductances are tracked online in order to adjust the current references thus achieving a higher control accuracy. In [30], a FOC control scheme is proposed, where the PI control parameters are continuously adapted to the actual system state, which improves the overall current dynamics.

An alternative to classical control approaches is the use of optimization-based control techniques such as model predictive control (MPC). When using MPC, a parametric optimization problem is formulated that exploits a model of the plant to be controlled and enforces constraints while minimizing a certain objective function. Although MPC can in principle improve the control performance and ease the controller design [24], meeting the required sampling times is in general a challenging task due to the high computational burden associated with the solution of the underlying optimization problems.

In order to circumvent this difficulty, several algorithmic strategies have been proposed over the past decade that use different approaches and (potentially) different formulations of the optimal control problems to be solved. Among the possible classifications of methods present in the literature, in the fields of electrical drives and power electronics, a fundamental distinction can be made between what is sometimes referred to as finite (FS-) and continuous control set (CS-) MPC [43], [8].

In FS-MPC, the switch positions of the power converter are regarded as optimization variables leading to mixed-integer programs. In this way, the need for an external modulator is eliminated and the switching sequences are directly determined by the solution to the optimal control problem (hence the name "direct" MPC used in some of the literature on MPC for electrical drives and power converters [23]). 


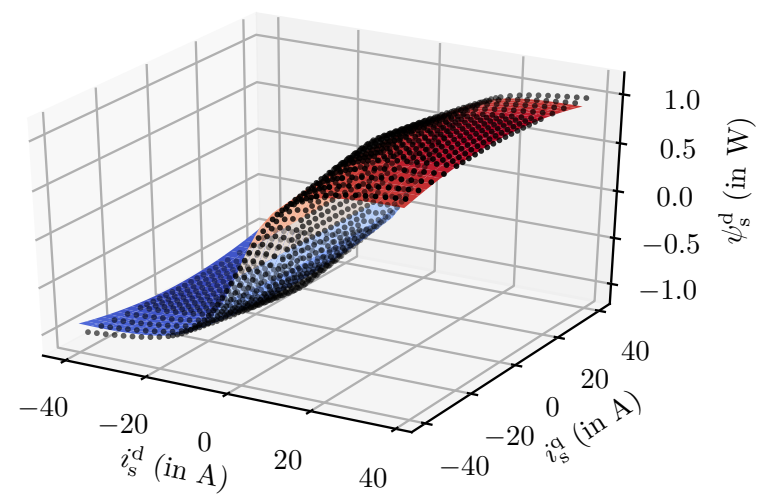

(a) fitted grey box flux model - d-component

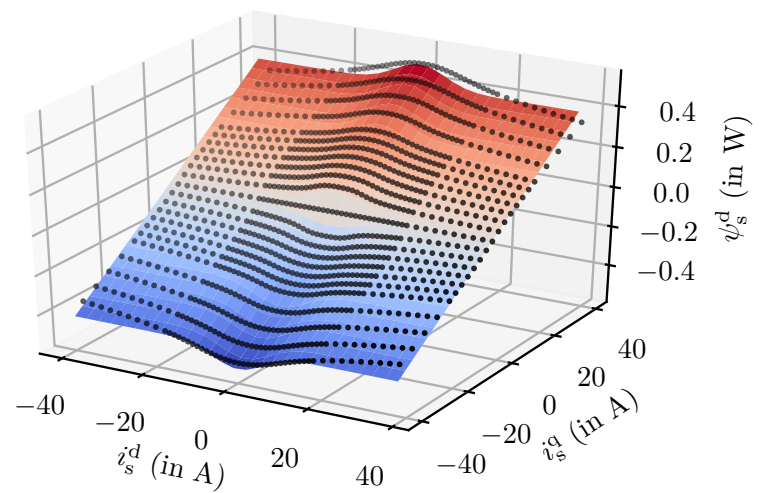

(b) fitted grey box flux model - q-component

Figure 1: Nonlinear flux linkage of a real RSM obtained from FEM data $\hat{\Psi}_{\mathrm{s}}^{\mathrm{d}}$ (solid surface) and fitted grey box model $\Psi_{\mathrm{s}}^{\mathrm{d}}$ (dotted). The worst-case relative error amounts to less than $10 \%$.

When using CS-MPC instead, we delegate the determination of switching sequences to an external modulator in order to obtain a continuous optimization problem. For this reason, CS-MPC is sometimes referred to as "indirect" MPC [23]. Although the computation times associated with this latter approach scale favourably with prediction horizon length and number of control variables (typically complexity $\mathcal{O}(N) n_{u}+$ $\left.n_{x}\right)^{3}$ ) can be achieved, where $N, n_{u}$ and $n_{x}$ represent horizon length, number of inputs and states, respectively), for short horizons, strategies based, e.g., on sphere decoding algorithms applied to FS-MPC formulations can achieve sufficiently short computation times. On the contrary, CS-MPC is generally regarded as more computationally expensive and it is still, arguably for this reason, largely unexplored [23].

Among the experimental results in the literature obtained with CS-MPC, in [2] a DC-excited synchronous motor is controlled using the real-time iteration method. In [16], a fixed-point iteration scheme is used to control a permanent magnet synchronous machine. Among applications leveraging linear-quadratic CS-MPC we mention the work in [14] in which permanent magnet synchronous machines and induction machines are controlled using explicit model predictive control. Finally, in the recent work in [7], an active-set algorithm is used to solve the convex QPs arising from a linear-quadratic CS-MPC formulation to control a PMSM.

\section{A. Contribution}

In this paper, we describe the design and implementation details together with simulation and experimental results of a nonlinear CS-MPC controller (CS-NMPC) for an RSM. The contributions of the present work are:

- We describe the design and implementation details of a tracking CS-NMPC formulation that relies on the software package acados, which is capable of achieving timings in the microsecond time scale necessary to control the electrical drive.

- We propose the use of a simple grey box model for the flux maps of RSMs with a small number of parameters that can be used for online applications where computation times are of key importance.
- Finally, we present simulation and experimental results that confirm the validity of the proposed control formulation and its implementation and its superior performance in comparison with state-of-the-art methods from the field of classical control. This is, to the best of the authors' knowledge, one the of the earliest experimentally validated applications of CS-NMPC to an RSM.

\section{BACKGROUND ON RSMS AND NMPC}

In order to facilitate the discussion of the design and implementation of the proposed controller, in the following, mathematical models of RSMs and voltage source inverters (VSI) will be derived and numerical methods for NMPC will be introduced. Note that the argument $(t)$, used to denote dependence on time, is sometimes dropped for the sake of readability. Moreover, we use the notation $\|x\|_{P}=\sqrt{x^{\top} P x}$, for some positive definite matrix $P$, to denote the $P$-weighted Euclidean norm of the vector $x$.

\section{A. Generic model of the RSM}

The machine model in the synchronously rotating $(\mathrm{d}, \mathrm{q})$ reference frame is given by [28, Chap. 14]

$$
\begin{gathered}
u_{\mathrm{s}}=R_{\mathrm{s}} i_{\mathrm{s}}+\omega \overbrace{\left[\begin{array}{cc}
0 & -1 \\
1 & 0
\end{array}\right]}^{=: J} \psi_{\mathrm{s}}\left(i_{\mathrm{s}}\right)+\frac{\mathrm{d}}{\mathrm{d} t} \psi_{\mathrm{s}}\left(i_{\mathrm{s}}\right), \\
\frac{\mathrm{d}}{\mathrm{d} t} \omega=\frac{n_{\mathrm{p}}}{\Theta}\left[m_{\mathrm{m}}\left(i_{\mathrm{s}}\right)-m_{\mathrm{l}}\right], \quad \frac{\mathrm{d}}{\mathrm{d} t} \phi=\omega,
\end{gathered}
$$

where $u_{\mathrm{s}}:=\left(u_{\mathrm{s}}^{\mathrm{d}}, u_{\mathrm{s}}^{\mathrm{q}}\right)^{\top}$ are the applied stator voltages, $R_{\mathrm{s}}$ is the stator resistance, $i_{\mathrm{s}}:=\left(i_{\mathrm{s}}^{\mathrm{d}}, i_{\mathrm{s}}^{\mathrm{q}}\right)^{\top}$ are the stator currents and $\psi_{\mathrm{s}}:=\left(\psi_{\mathrm{s}}^{\mathrm{d}}, \psi_{\mathrm{s}}^{\mathrm{q}}\right)^{\top}$ are the stator flux linkages (functions of $\left.i_{\mathrm{s}}\right)$. The $(\mathrm{d}, \mathrm{q})$-reference frame rotates with electrical angular velocity $\omega=n_{\mathrm{p}} \omega_{\mathrm{m}}$ of the rotor, where $n_{\mathrm{p}}$ is the number of pole pairs and $\omega_{\mathrm{m}}$ denotes the mechanical angular velocity of the machine. Furthermore, $\Theta$ is the total moment of inertia,

$$
m_{\mathrm{m}}\left(i_{\mathrm{s}}\right):=\frac{3}{2} n_{\mathrm{p}}\left(i_{\mathrm{s}}\right)^{\top} J \psi_{\mathrm{s}}\left(i_{\mathrm{s}}\right)
$$

is the electro-magnetic machine torque, and $m_{1}$ represents an external (time-varying) bounded load torque. 
In order to formulate an optimal control problem, the flux dynamics can be described, based on (1), by the following differential algebraic equation (DAE):

$$
\begin{aligned}
\frac{\mathrm{d}}{\mathrm{d} t} \psi_{\mathrm{s}} & =u_{\mathrm{s}}-R_{\mathrm{s}} i_{\mathrm{s}}-\omega J \psi_{\mathrm{s}}+v, \\
0 & =\psi_{\mathrm{s}}-\Psi_{\mathrm{s}}\left(i_{\mathrm{s}}\right),
\end{aligned}
$$

where $\Psi_{\mathrm{s}}:=\left(\Psi_{\mathrm{s}}^{\mathrm{d}}, \Psi_{\mathrm{s}}^{\mathrm{q}}\right)^{\top}: \mathbb{R}^{2} \rightarrow \mathbb{R}^{2}$ defines the algebraic constraints based on the identified flux maps and $v:=\left(v^{\mathrm{d}}, v^{\mathrm{q}}\right)^{\top}$ are additive disturbances which will be used in an offset-free NMPC setting (see Section II-F).

Based on the available flux maps computed through the finite element method (FEM), we obtained a continuously differentiable model by fitting a simple grey box model. Due to their low number of parameters and simple structure, we propose the following parametrization of the flux maps:

$$
\begin{aligned}
& \Psi_{\mathrm{s}}^{\mathrm{d}}\left(i_{\mathrm{s}}^{\mathrm{d}}, i_{\mathrm{s}}^{\mathrm{q}}, \theta_{\mathrm{d}}\right)= \\
& \frac{c_{0}^{\mathrm{d}}}{\sqrt{2 \pi \sigma_{\mathrm{q}}^{2}}} \exp \left(-\gamma\left(i_{\mathrm{s}}^{\mathrm{q}}, \sigma_{\mathrm{q}}\right)\right) \operatorname{atan}\left(c_{1}^{\mathrm{d}} i_{\mathrm{s}}^{\mathrm{d}}\right)+c_{2}^{\mathrm{d}} i_{\mathrm{s}}^{\mathrm{d}}
\end{aligned}
$$

and

$$
\begin{aligned}
& \Psi_{\mathrm{s}}^{\mathrm{q}}\left(i_{\mathrm{s}}^{\mathrm{d}}, i_{\mathrm{s}}^{\mathrm{q}}, \theta_{\mathrm{q}}\right)= \\
& \quad \frac{c_{0}^{\mathrm{q}}}{\sqrt{2 \pi \sigma_{\mathrm{d}}^{2}}} \exp \left(-\gamma\left(i_{\mathrm{s}}^{\mathrm{d}}, \sigma_{\mathrm{d}}\right)\right) \operatorname{atan}\left(c_{1}^{\mathrm{q}} i_{\mathrm{s}}^{\mathrm{q}}\right)+c_{2}^{\mathrm{q}} i_{\mathrm{s}}^{\mathrm{q}},
\end{aligned}
$$

with

$$
\gamma(x, y):=\frac{1}{2}\left(\frac{x}{y}\right)^{2}
$$

and where the unknown parameters involved are

$$
\theta_{\mathrm{d}}:=\left(c_{0}^{\mathrm{d}}, c_{1}^{\mathrm{d}}, c_{2}^{\mathrm{d}}, \sigma_{\mathrm{d}}\right)
$$

and

$$
\theta_{\mathrm{q}}:=\left(c_{0}^{\mathrm{q}}, c_{1}^{\mathrm{q}}, c_{2}^{\mathrm{q}}, \sigma_{\mathrm{q}}\right)
$$

This parametrization of the flux maps is, to the authors' best knowledge, novel and it is able to capture the main features of the flux maps with only 4 parameters per flux component. The numerical values of the coefficients can be computed by solving the following (decoupled) nonlinear least-squares problems:

$$
\begin{aligned}
& \min _{\theta_{\mathrm{d}}} \sum_{j=1}^{m} \sum_{k=1}^{n}\left(\Psi_{\mathrm{s}}^{\mathrm{d}}\left(\bar{i}_{\mathrm{s}, j}^{\mathrm{d}}, \bar{i}_{\mathrm{s}, k}^{\mathrm{q}}, \theta_{\mathrm{d}}\right)-\hat{\Psi}_{\mathrm{s}}^{\mathrm{d}}\left(\bar{i}_{\mathrm{s}, j}^{\mathrm{d}}, \bar{i}_{\mathrm{s}, k}^{\mathrm{q}}\right)\right)^{2} \\
& \min _{\theta_{\mathrm{q}}} \sum_{j=1}^{m} \sum_{k=1}^{n}\left(\Psi_{\mathrm{s}}^{\mathrm{q}}\left(\bar{i}_{\mathrm{s}, j}^{\mathrm{d}}, \bar{i}_{\mathrm{s}, k}^{\mathrm{q}}, \theta_{\mathrm{q}}\right)-\hat{\Psi}_{\mathrm{s}}^{\mathrm{q}}\left(\bar{i}_{\mathrm{s}, j}^{\mathrm{d}}, \bar{i}_{\mathrm{s}, k}^{\mathrm{q}}\right)\right)^{2}
\end{aligned}
$$

where $\bar{i}_{\mathrm{s}, j}^{\mathrm{d}}$ and $\bar{i}_{\mathrm{s}, k}^{\mathrm{q}}$ are the $j$-th and $k$-th current data points associated with the flux values $\hat{\Psi}_{\mathrm{s}}^{\mathrm{d}}$ and $\hat{\Psi}_{\mathrm{s}}^{\mathrm{q}}$ obtained from FEM analysis. The fitting problems have been solved with the MATLAB Curve Fitting Toolbox and the resulting fitted model is shown in Figure 1

\section{B. Model of the two-level VSI}

The machine is supplied by a two-level voltage source inverter (VSI), which - on average over one switching period $T_{\mathrm{s}}$ - translates a given voltage reference

$$
u_{\mathrm{s}, \mathrm{ref}}^{\mathrm{s}}:=\left(u_{s, \mathrm{ref}}^{\alpha}, u_{s, \mathrm{ref}}^{\beta}\right)
$$

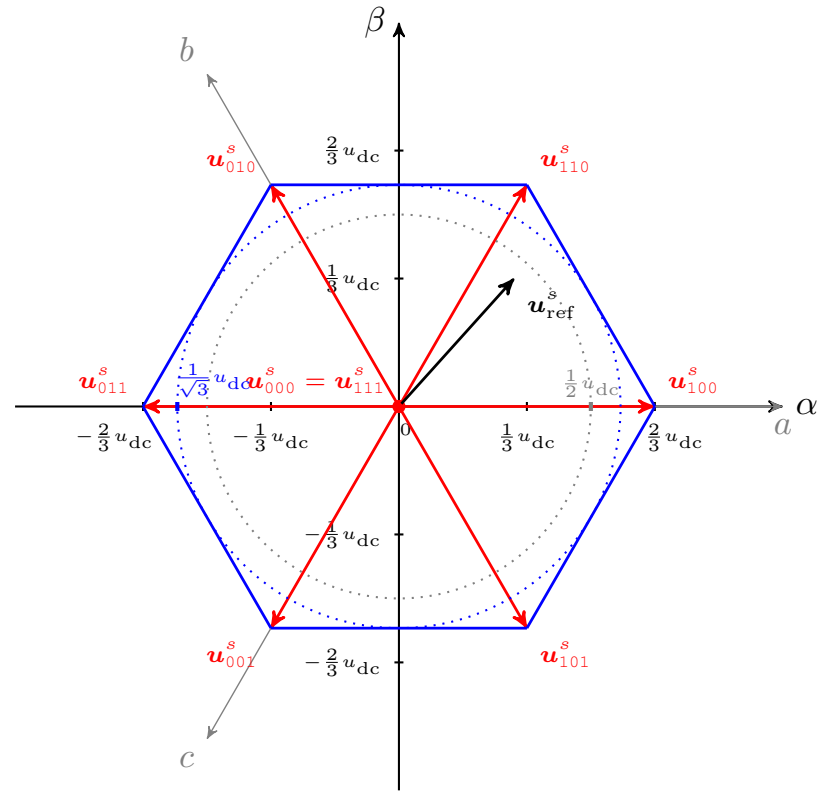

Figure 2: Voltage hexagon associated with the two-level VSI.

(in the stationary $s=(\alpha, \beta)$-reference frame) into the inverter output voltage $u_{\mathrm{s}}^{\mathrm{s}}$, i.e.

$$
u_{\mathrm{s}}^{\mathrm{s}}\left(k T_{\mathrm{s}}\right) \approx u_{\mathrm{s}, \mathrm{ref}}^{\mathrm{s}}\left((k-1) T_{\mathrm{s}}\right), \quad k \in \mathbb{N} .
$$

Since a two-level voltage source inverter may produce a total of eight unique switching vectors, i.e. $s_{\mathrm{s}}^{a b c}:=\left(s_{\mathrm{s}}^{\mathrm{a}}, s_{\mathrm{s}}^{\mathrm{b}}, s_{\mathrm{s}}^{\mathrm{c}}\right)^{\top} \in$ $\{000,001,010,100,011,101,110,111\}$, the typical voltage hexagon in the $\alpha \beta$-plane is obtained (see Figure 2), where

$$
u_{\mathrm{s}}^{\mathrm{S}}=\kappa u_{\mathrm{dc}}\left[\begin{array}{ccc}
\frac{1}{2} & 0 & -\frac{1}{2} \\
0 & \frac{\sqrt{3}}{2} & 0
\end{array}\right]\left[\begin{array}{ccc}
1 & -1 & 0 \\
0 & 1 & -1 \\
-1 & 0 & 1
\end{array}\right] s_{\mathrm{s}}^{a b c}
$$

depends on the switching vector $s_{\mathrm{s}}^{a b c}$ and the Clarke-factor $\kappa \in$ $\{2 / 3, \sqrt{2 / 3}\}$ [28, Chap. 14]. Using space-vector modulation (SVM) to generate the switching vector, any voltage reference within the circle of radius $u_{\mathrm{dc}} / \sqrt{3}$ can be realized, with $u_{\mathrm{dc}}$ denoting the (assumed constant) DC-link voltage. Finally, the inverter output voltage is transformed into the rotating $(\mathrm{d}, \mathrm{q})$ reference frame using the inverse Park transformation, i.e.

$$
u_{\mathrm{s}}=\left[\begin{array}{c}
u_{\mathrm{s}}^{\mathrm{d}} \\
u_{\mathrm{s}}^{\mathrm{q}}
\end{array}\right]=\underbrace{\left[\begin{array}{cc}
\cos (\phi) & \sin (\phi) \\
-\sin (\phi) & \cos (\phi)
\end{array}\right]}_{=: T_{\mathrm{p}}(\phi)^{-1}} u_{\mathrm{s}}^{\mathrm{s}}
$$

From now on, since we will only refer to currents, fluxes and voltages applied to the stator and in the (d-q)-frame, we will simplify the notation by dropping the associated subscript such that, for example, $i=\left(i_{\mathrm{s}}^{\mathrm{d}}, i_{\mathrm{s}}^{\mathrm{q}}\right)$ denotes the stator currents in the (d-q)-frame.

\section{Nonlinear model predictive control}

NMPC is an optimization-based control strategy that allows one to tackle control problems involving potentially nonlinear dynamics, constraints and objectives by solving online a series of parametric nonlinear programs (NLP). Due to the computational challenge of solving NLPs within the required 


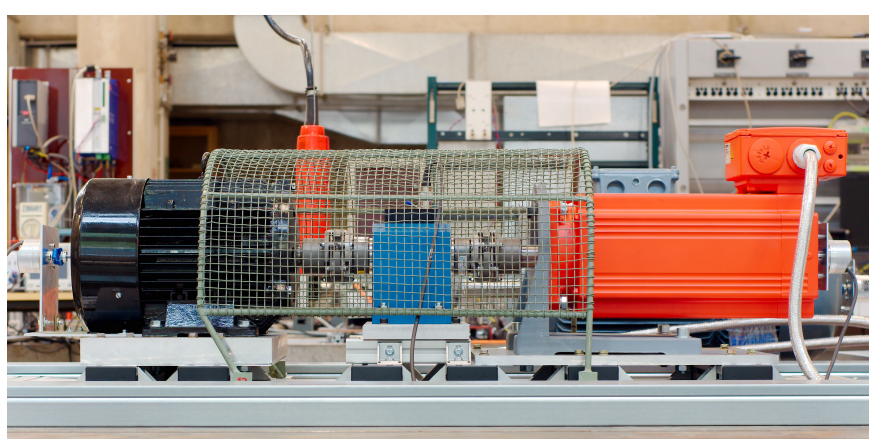

Figure 3: Laboratory setup with $\mathrm{dSPACE}$ real-time system, voltage-source inverters connected back-to-back, RSM, PMSM and torque sensor.

sampling times, NMPC has initially found application in the chemical industry and in the field of process control in general [49], where relatively slow dynamics allow for sufficiently long sampling times. In more recent times, due to the development of increasingly efficient numerical methods and software implementation and due to the growing computational power of embedded control units, NMPC has gradually become a viable approach for applications with much shorter computation times. Among other recent works that reported on the successful application of MPC to control systems with sampling times in the range of milli- and microsecond we mention [1, 55].

In this paper, we will regard the following standard tracking formulation with prediction horizon $T_{h}$ and $N$ shooting nodes, where the squared deviation of fluxes $\psi$ and voltages $u$ from properly defined steady-state references are penalized:

$$
\begin{aligned}
& \min _{\substack{\psi_{0}, \ldots, \psi_{N} \\
u_{0}, \ldots, u_{N-1}}} \frac{T_{h}}{2 N} \sum_{i=0}^{N-1}\left\|\begin{array}{l}
\psi_{i}-\bar{\psi} \\
u_{i}-\bar{u}
\end{array}\right\|_{W}^{2}+\frac{1}{2}\left\|\psi_{N}-\bar{\psi}\right\|_{W_{N}}^{2} \\
& \text { s.t. } \quad \psi_{0}-\psi_{\mathrm{e}}=0 \text {, } \\
& g\left(\psi_{i}, u_{i}, \omega_{\mathrm{e}}, v_{\mathrm{e}}\right)-\psi_{i+1}=0, i=0, \ldots, N-1, \\
& u_{i}^{\top} u_{i} \leq\left(\frac{u_{\mathrm{dc}}}{\sqrt{3}}\right)^{2}, \quad i=0, \ldots, N-1, \\
& \hat{C} u_{i} \leq \hat{c}, \quad i=0, \ldots, N-1,
\end{aligned}
$$

where $g$ describes the discretized dynamics obtained by integrating the differential-algebraic model in (3) using the GaussLegendre collocation method of order 2 assuming constant (estimated) angular velocity $\omega_{\mathrm{e}}$ and disturbances $v_{\mathrm{e}}$. The variables $\bar{\psi}$ and $\bar{u}$ denote the steady-state references computed for a given desired torque using a maximum-torque-per-Ampere (MTPA) criterion [15]. Given the flux maps obtained from FEM data in Figure 1 it is possible to compute off-line lookup tables (LUTs) that contain the MTPA reference fluxes and voltages for a finite number of values of the target torque in a specified range. The LUTs are then interpolated online in order to compute approximate values of $\bar{\psi}$ and $\bar{u}$ associated with the specified target torque $\bar{m}$ (see Figure 4 ).

The convex quadratic constraint in (14) describes the circular input feasible set introduced in Section II-B. Finally, $\hat{C}$ and $\hat{c}$ define polytopic constraints (which we will later refer to as "safety" constraints) that are meant to be always inactive at any local solution of (14) (apart from a finite number of points

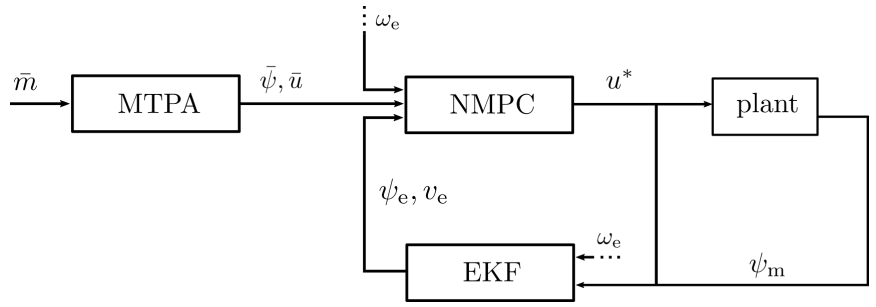

Figure 4: Control diagram: the MTPA LUTs provide the reference flux $\bar{\psi}$ and voltage $\bar{u}$ associated with a given reference torque $\bar{m}$. The NMPC controller computes the optimal control action based on the current state and disturbance estimate provided by an EKF.

where they are locally equivalent to the linearized spherical constraint), but can mitigate constraint violation of intermediate SQP iterates. In particular, we define $\hat{C}$ and $\hat{c}$ such that the affine constraint defines an outer polytopic approximation with 6 facets as depicted in Figure 5. Notice that due to this formulation of the feasible set, linear independence constraint qualification can fail at a finite number of points where the linearization of the nonlinear constraint is equivalent to one of the affine constraints in (14). Although, this would violate a common assumption used in convergence theory for both $\mathrm{SQP}$ and some numerical methods for the solution of convex QPs, the active-set solver qPOASES that we employ for this application can handle redundant constraints through a strategy that determines which constraint needs to be removed from the working set [18].

Remark 1. Notice that the actual dynamics of the system involve a coupling of mechanical $(\omega)$ and electrical states $(\psi)$. It is however common, given the large difference between associated time constants, to assume a constant angular velocity $\omega$ when designing controllers. In our case, it allows us to use much shorter prediction horizons since we do not require the OCP in (14) to steer the speed of the motor to the desired reference, but only fluxes which directly map to currents and, for a given speed, to torques.

Problem (14) is used to define an implicit feedback policy that requires the solution of an instance of the parametric NLP at every sampling time, where the value of the parameter $\psi_{\mathrm{e}}$ is given by the current estimate of the system's state. The resulting solutions are feasible with respect to the constraints and minimize (at least locally) the cost function. Nominal and inherently robust stability of the closed-loop system can be guaranteed in a neighborhood of a steady-state by properly choosing the terminal cost [49].

Remark 2. Notice that formulations more general than (14) can in principle be used in the framework of NMPC. Among others, economic costs and more general nonlinear constraints and nonlinear cost terms, are features that can be included in the problem in order to better capture control design requirements. However, for the application discussed in this paper, the nonlinear least-squares problem described in (14) is general enough. 

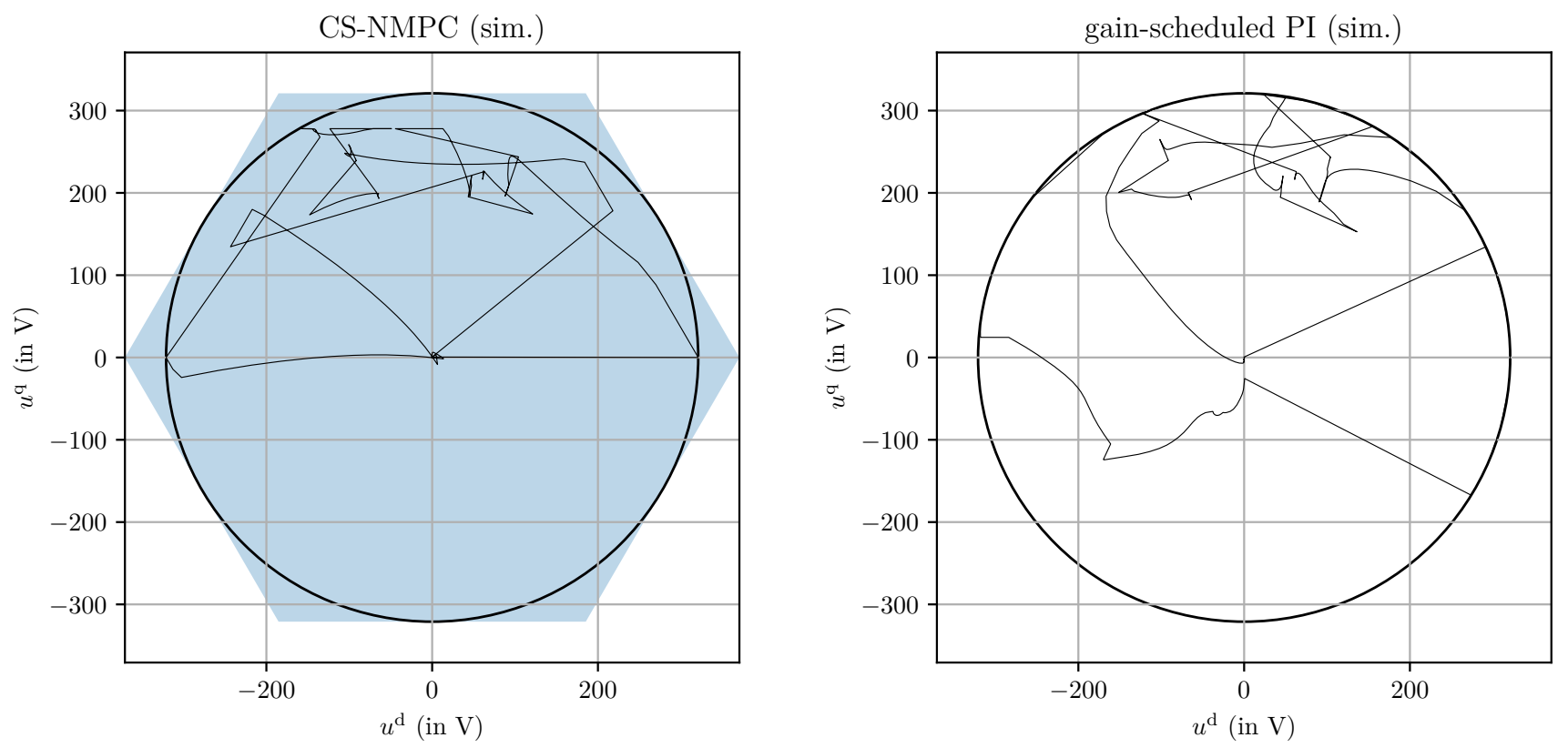

Figure 5: Current steps at $157 \frac{\mathrm{rad}}{\mathrm{s}}$ (simulation): results obtained using the CS-NMPC (left) and the gain-scheduled PI controller (right). The voltage spherical constraints are directly included into the control formulation using the SCQP strategy proposed in [52]. Additionally, a "safety" polytopic constraint is included which, due to its linearity, is always satisfied exactly.

\section{Numerical methods and software for NMPC}

In order to be able to solve problem (14) within the available computation time, the use of efficient numerical methods is fundamental. First, since (14) is obtained through a multipleshooting discretization strategy, an efficient way of computing evaluations of the discretized dynamics $g$ and of its firstorder (and potentially second-order) derivatives needs to be available. This is commonly achieved by means of numerical integration of the ordinary differential equation (ODE) or algebraic differential equations (DAE) describing the dynamics of the system. Although for ODEs explicit and implicit integration methods can be used, for DAEs, as for the system under consideration (see Section II-A), implicit schemes are generally necessary that involve the solution of nonlinear rootfinding problems via Newton-type iterations. In the context of NMPC, efficient strategies and tailored implementations are available that rely on, for example, exploiting the structure of the model [20, 44], on reuse of Jacobian factorizations and efficient sensitivity generation [47], on lifting-based formulations [45] and on inexact iterations [46].

Once the linearization is carried out, one generally needs to solve structured linear systems that can be used to compute the solution to a quadratic program $(\mathrm{QP})$ as in sequential quadratic programming (SQP), compute the update defined by an interior-point method or the one used by other various strategies such as, e.g., first-order methods. Since the description of the details of the different available approaches to solve (14) goes well beyond the scope of this work, we will focus, in the following, on the SQP strategy, which constitutes the basis for the real-time iteration (RTI) method used in this application.
When using SQP, a sequence of structured QPs of the following form needs to be solved:

$$
\begin{array}{cl}
\min _{\substack{s_{0}, \ldots, s_{N} \\
u_{0}, \ldots, u_{N-1}}} & \frac{1}{2} \sum_{i=0}^{N-1}\left[\begin{array}{c}
s_{i} \\
u_{i} \\
1
\end{array}\right]^{\top} H_{i}\left[\begin{array}{c}
s_{i} \\
u_{i} \\
1
\end{array}\right]+\frac{1}{2}\left[\begin{array}{c}
s_{N} \\
1
\end{array}\right]^{\top} H_{N}\left[\begin{array}{c}
s_{N} \\
1
\end{array}\right] \\
\text { s.t. } & s_{0}-x=0, \\
& s_{i+1}=A_{i} s_{i}+B_{i} u_{i}+c_{i}, i=0, \ldots, N-1, \\
& C_{i} u_{i}+D_{i} s_{i}+e_{i} \leq 0, \quad i=0, \ldots, N-1, \\
& D_{N} s_{N}+e_{N} \leq 0 .
\end{array}
$$

Here the parameter $x$ denotes the current state of the system and the states and inputs are denoted by $s \in \mathbb{R}^{n_{s}}$ and $u \in \mathbb{R}^{n_{u}}$, respectively. The matrices and vectors $A_{i} \in \mathbb{R}^{n_{s} \times n_{s}}$, $B_{i} \in \mathbb{R}^{n_{s} \times n_{u}}$ and $c_{i} \in \mathbb{R}^{n_{s}}$, for $i=0, \ldots, N-1$, define the linearized dynamics obtained through numerical integration and where $C_{i} \in \mathbb{R}^{n_{\pi} \times n_{u}}, D_{i} \in \mathbb{R}^{n_{\pi} \times n_{s}}, e_{i} \in \mathbb{R}^{n_{\pi}}$, for $i=0, \ldots, N-1$, and $D_{N} \in \mathbb{R}^{n_{\pi_{N}} \times n_{s}}, e_{N} \in \mathbb{R}^{n_{\pi_{N}}}$ define the linearized constraints. Finally the matrices

$$
H_{i}=\left[\begin{array}{ccc}
Q_{i} & S_{i} & q_{i} \\
S_{i}^{\top} & R_{i} & r_{i} \\
q_{i}^{\top} & r_{i}^{\top} & 0
\end{array}\right] \quad \text { and } \quad H_{N}=\left[\begin{array}{cc}
Q_{N} & q_{N} \\
q_{N}^{\top} & 0
\end{array}\right] \text {, }
$$

with $Q_{i} \in \mathbb{R}^{n_{s} \times n_{s}}, S_{i} \in \mathbb{R}^{n_{s} \times n_{u}}, R_{i} \in \mathbb{R}^{n_{u} \times n_{u}}, r_{i} \in \mathbb{R}^{n_{u}}$, $q_{i} \in \mathbb{R}^{n_{s}}$, for $i=0, \ldots, N-1$, and $Q_{N}, q_{N}$ define the cost of the QP. The matrices and vectors defining the QP (15) are computed based on the linearization associated with the current primal-dual iterate $z=(s, u, \lambda \mu)$ (where $\lambda$ and $\mu$ represent the Lagrange multipliers associated with the equality and inequality constraints in (14), respectively) and, after solving (15), the iterate is updated, i.e., $z_{+} \leftarrow z+\alpha \Delta z$, where 
$\Delta z$ represents the primal-dual step associated with the solution of the QP constructed at the linearization point $z$ and $\alpha>0$ is the step size, which can be adjusted to achieve convergence. Under standard assumptions [40], the iterates converge to a local minimum of (14).

Due to the computational burden associated with the solution of the QPs and re-linearization of the original NLP in (14), several approximate strategies can be used that can significantly reduce computation times (e.g., [56], [25], [17]). In this work, we will use the RTI strategy [9, 10], which relies on a single SQP iteration in order to provide an approximate feedback law. Attractivity properties of the combined systemoptimizer dynamics associated with the RTI strategy have been first analyzed in [12] and [11] for a simplified setting without inequality constraints and the recent work in [59] and [58] extends these results proving asymptotic stability for the inequality constrained case too. The RTI has been implemented in the software packages MUSCOD-II [13], ACADO [31] and in its successor acados [51], which is shortly described in the next subsection.

\section{E. The acados framework}

The high-performance software package acados [51] provides a modular framework for NMPC and moving horizon estimation (MHE). It consists of a $\mathrm{C}$ library that implements building blocks needed to solve NLPs arising from NMPC and MHE formulations. It relies on the high-performance linear algebra package BLASFEO [21] and on the quadratic program (QP) solver HPIPM [22] and contains efficient implementations of explicit and implicit integration methods. Moreover, it interfaces a number of QP solvers such as gPOASES [19] and OSQP [50] and it provides high-level Python and MATLAB interfaces. Through these interfaces, one can conveniently specify optimal control problems and code-generate a selfcontained C library that implements the desired solver and can be easily deployed onto embedded control units such as dSPACE using the automatically generated $\mathrm{C}$ wrapper and $\mathrm{S}$ Function. The code-generation takes place through templated $\mathrm{C}$ code which is rendered by the Tera templating engine written in Rust. In this way, human-readable $\mathrm{C}$ code can be generated that facilitates the deployment on the target hardware.

\section{F. NMPC offset-free tracking formulation}

In order to achieve offset-free regulation, we adopt the standard strategies discussed, for example, in [42]. In particular, we use the following augmented dynamics to design an extended Kalman filter (EKF):

$$
\begin{aligned}
\frac{\mathrm{d}}{\mathrm{d} t} \psi & =u-R i-\omega J \psi+v, \\
\frac{\mathrm{d}}{\mathrm{d} t} v & =0, \\
0 & =\psi-\Psi(i),
\end{aligned}
$$

where the disturbance state $v$ is introduced and we assume that pseudo-measurements $\psi_{\text {meas }}$ are available through the interpolated FEM flux maps:

$$
\psi_{\text {meas }}=\hat{\Psi}\left(i_{\text {meas }}\right),
$$

while current measurements $i_{\text {meas }}$ are physically carried out on the machine. A standard EKF is designed using (17) and (18) which uses flux measurements to estimate fluxes $\psi_{\mathrm{e}}$ and disturbances $v_{\mathrm{e}}$. Notice that the angular velocity $\omega_{\mathrm{e}}$ is estimated externally and is considered as a constant-overtime parameter that is updated at every sampling time. In [41. Theorem 14] a streamlined version of the results from [38, 39, 42] is presented, where under the assumptions, among others, of observability of the augmented dynamics (17) and asymptotically constant disturbances $v$, the steady-state of the closed-loop system can be proved to be offset-free.

\section{IMPLEMENTATION AND SIMULATION RESULTS}

An RTI strategy [10], where a single QP of an SQP algorithm is carried out per sampling time, is used to solve (14). In particular, the generalized Gauss-Newton Hessian approximation proposed in [51] is used. In this way, the (positive) curvature contribution due to the convex spherical constraints on voltages can be exploited in order to improve the Hessian approximation used in the QP subproblems. Although in our experience this improves a lot the convergence of the RTI iterates on this specific problem, the approximate feedback law can, from time to time, be largely infeasible with respect to the nonlinear spherical constraints (recall that the intermediate full-step SQP iterates are feasible only with respect to linear constraints). Since a-posteriori projection of the control actions onto the feasible set can largely deteriorate the control performance, we add extra polytopic "safety" constraints (defined by $\hat{C}$ and $\hat{c}$ in (14)) around the spherical ones in order to ensure that the constraint violation is bounded at any successfully computed iterate.

In order to be able to meet the short sampling times required to control the electrical drive, we use a prediction horizon of $T_{h}=3.2 \mathrm{~ms}$ obtained with 2 shooting nodes $(N=2)$ and we use the QP solver qPOASES, which is particularly suited for problems with short horizons [33]. For both simulation and experimental results the controller is run at $4 \mathrm{kHz}$.

Remark 3. Notice that the discretization time $\left(T_{h} / 2=\right.$ $1.6 \mathrm{~ms})$ used in the optimal control problem is not equivalent to the sampling time $T_{s}=0.25 \mathrm{~ms}$. This setting, which we might call "oversampled" NMPC, allows one to obtain a longer prediction horizon without increasing the number of optimization variables. Although a theoretical analysis of this strategy is well beyond the scope of this paper, we point the interested reader to [26] where it is shown that fundamental properties of the feedback policy hold for the oversampled setting. Moreover, notice that this setting is used in [59] and [58] in order to prove asymptotic stability of the combined system-optimizer dynamics.

We have tuned the weights in 14 until satisfactory closedloop performance could be achieved in simulation resulting in $W=\operatorname{blkdiag}\left(312.5 \cdot \mathbb{I}_{2}, 1 e^{-4} \cdot \mathbb{I}_{2}\right)$ and $W_{N}$ set to the corresponding LQR cost obtained with the dynamics linearized at $i=0, \psi=0, u=0$ and $\omega_{\mathrm{m}}=0$. Although the terminal cost should in general be updated together with the desired reference, a fixed terminal cost was able to provide satisfactory control performance. 

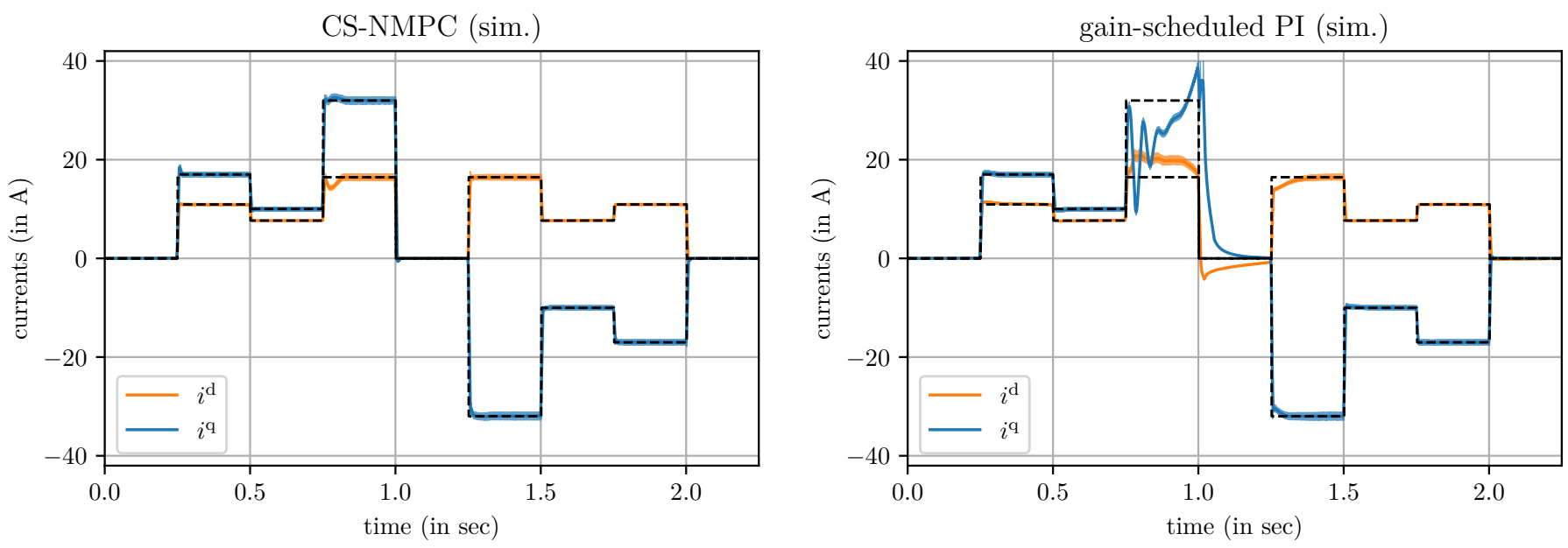

Figure 6: Current steps at $157 \frac{\mathrm{rad}}{\mathrm{s}}$ (simulation): results obtained using the CS-NMPC (left) and gain-scheduled PI controller (right). The CS-NMPC controller outperforms the PI controller, especially when the input constraints become active (e.g., between $t=0.75 \mathrm{~s}$ and $t=1.00 \mathrm{~s}$ ). At the same time, a faster transient can be achieved even when the constraints become active only for a short time.
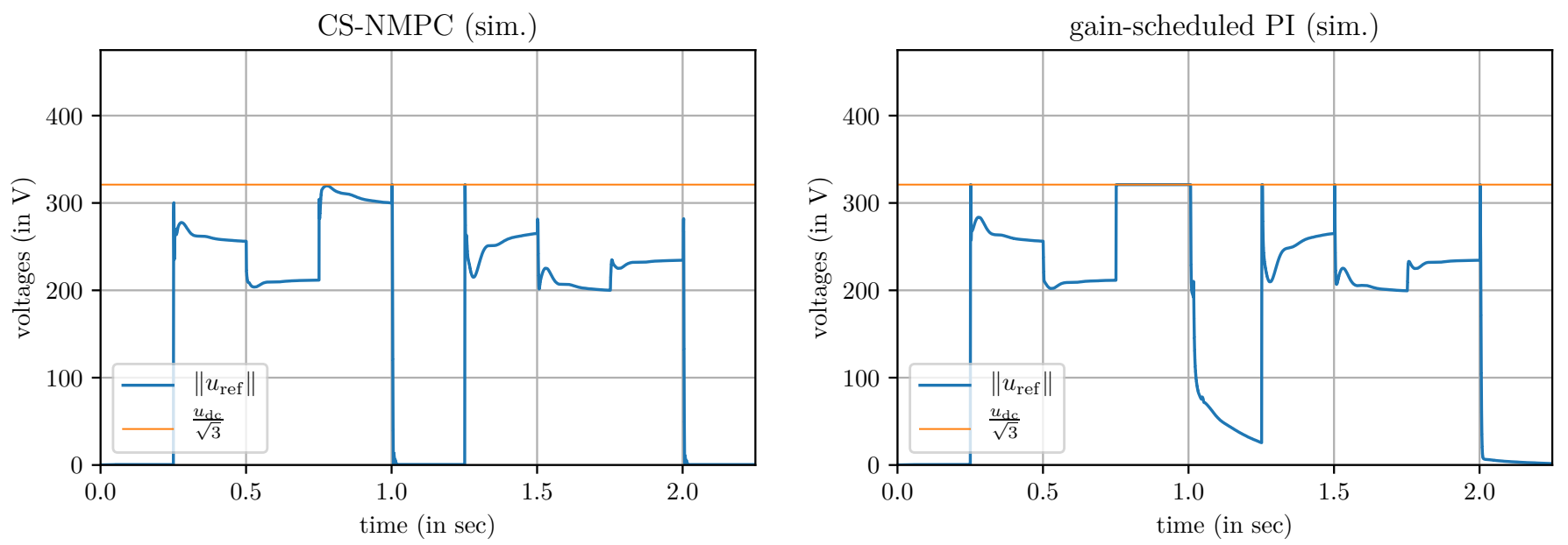

Figure 7: Current steps at $157 \frac{\mathrm{rad}}{\mathrm{s}}$ (simulation): two-norm of voltage references $u_{\mathrm{ref}}$ commanded by the two controllers and $u_{\mathrm{dc}}$ over time in simulation at $157 \frac{\mathrm{rad}}{\mathrm{s}}$. During the third current step, the PI controller saturates and does not steer the system to the desired reference. Notice that the input commanded by the PI controller remains saturated during the entire step. On the contrary, the CS-NMPC controller, after an initial saturation, steers the current to the (feasible) reference values.

In the following, we discuss simulation and experimental results obtained using the above described RTI strategy to solve (14) with acados.

In order to validate, first in simulation and then experimentally, the proposed approach, we regard a setting where the RSM is connected to a permanent synchronous machine (PMSM) which can be used to simulate different load conditions. The CS-NMPC controller has been implemented in acados using its Python interface and integrated in a Simulink model that makes use of a high-fidelity model of the system to be controlled including a model of the PMSM and of the two-level VSI described in Section III Moreover, we have implemented an EKF based on the augmented model (17) using the implicit integrators available in acados.

We set the PMSM's controller such that it maintains a constant rotational speed and we change the torque reference fed to the RSM's controller to assess the tracking performance of the proposed controller. We compare the closedloop trajectories obtained with the ones achieved when using instead the gain-scheduled PI controller with anti-windup presented in [29]. The parameter tuning used in [29] was used as baseline and we adapted the parameters until the PI controller was able to stabilize the system and achieve satisfactory control performance for the scenario under analysis. In particular, we had to scale down the proportional and integral coefficients by a factor two. For the sake of reproducibility the entire simulation environment is made available at https://github.com/zanellia/cs_nmpc_rsm.

In order to highlight the advantages of using a controller which can handle constraints directly, we set the reference 

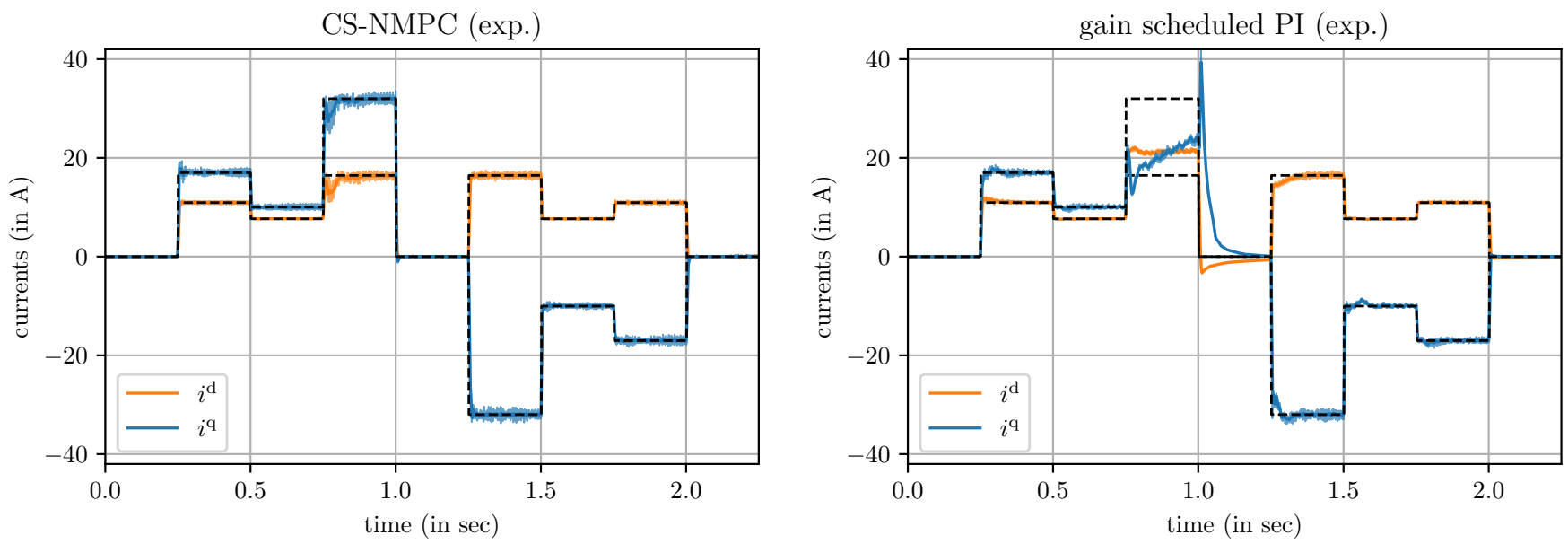

Figure 8: Current steps at $157 \frac{\mathrm{rad}}{\mathrm{s}}$ (experiment): results obtained using the proposed CS-NMPC controller (left) and gain-scheduled PI controller (right). The CS-NMPC controller outperforms the PI controller, especially when the input constraints become active (e.g., between $t=0.75 \mathrm{~s}$ and $t=1.00 \mathrm{~s}$ ). At the same time, as it can be seen especially between $t=1.25 \mathrm{~s}$ and $t=1.50 \mathrm{~s}$, a faster transient can be achieved, even when the constraints are active only for a short time.
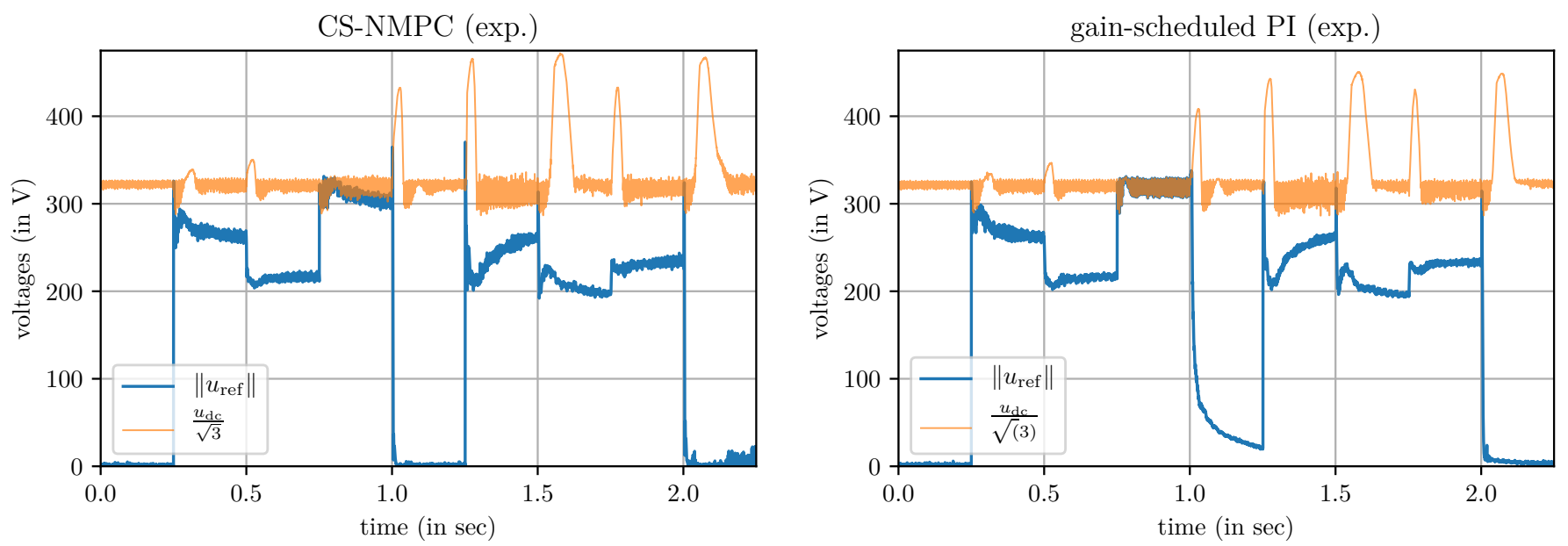

Figure 9: Current steps at $157 \frac{\mathrm{rad}}{\mathrm{s}}$ (experiment): two-norm of measured voltage references $u_{\text {ref }}$ commanded by the two controllers and $u_{\mathrm{dc}}$ over time. During the third current step, the PI controller saturates and does not steer the system to the desired reference. Notice that the input commanded by the PI controller remains saturated during the entire step. On the contrary, the CS-NMPC controller, after an initial saturation, steers the current to the (feasible) reference values.

speed to a value that is close to the limit value $\omega_{\mathrm{m}}^{\star}$ computed as follows:

$$
\begin{aligned}
\omega_{\mathrm{m}}^{\star}:=\frac{1}{2} \arg \max _{\omega} & \omega \\
\text { s.t. } & \left\|R_{s} i_{\text {ref }}+\omega J \psi_{\text {ref }}\right\|_{2} \leq\left(\frac{u_{\mathrm{dc}}}{\sqrt{3}}\right) .
\end{aligned}
$$

Since the optimal value is achieved at the boundaries of the feasible set, we can simply solve for $\omega$ the quadratic equation

$$
\left\|R_{s} i_{\mathrm{ref}}+\omega J \psi_{\mathrm{ref}}\right\|_{2}^{2}-\left(\frac{u_{\mathrm{dc}}}{\sqrt{3}}\right)^{2}=0
$$

such that, for the values $i_{\text {ref }}=(16.45,31.99) \mathrm{A}$ and $\psi_{\text {ref }}=$ $(0.819,0.417) \mathrm{Wb}$ associated with the torque value $58 \mathrm{~N} \mathrm{~m}$ and $u_{\mathrm{dc}}=556 \mathrm{~V}$, we obtain $\omega_{\mathrm{m}}^{\star}=169.32 \frac{\mathrm{rad}}{\mathrm{s}}$. Hence, we set $\omega_{\mathrm{m}, \text { ref }}=157 \frac{\mathrm{rad}}{\mathrm{s}} \approx \omega_{\mathrm{m}, \text { nom }}$ for both the simulation and experimental scenarios. Finally, the parameters used in the simulations match the ones of the physical setup and are reported in Table I] The current trajectories obtained with the CS-NMPC and PI controller are reported in Figure 6 (similarly for input trajectories in Figure 7). It is clear from the plots that the tracking performance achieved by the CSNMPC controller is largely superior to the one obtained by the PI controller, especially when the input constraints become active (e.g., between $t=0.75 \mathrm{~s}$ and $t=1.00 \mathrm{~s}$ ). At the same time, as it can be seen from the current trajectories in Figure 7 between $t=1.25 \mathrm{~s}$ and $t=1.50 \mathrm{~s}$, a faster transient can be achieved, even when the constraints are active only for a short time. In Appendix A we report additional results obtained with 


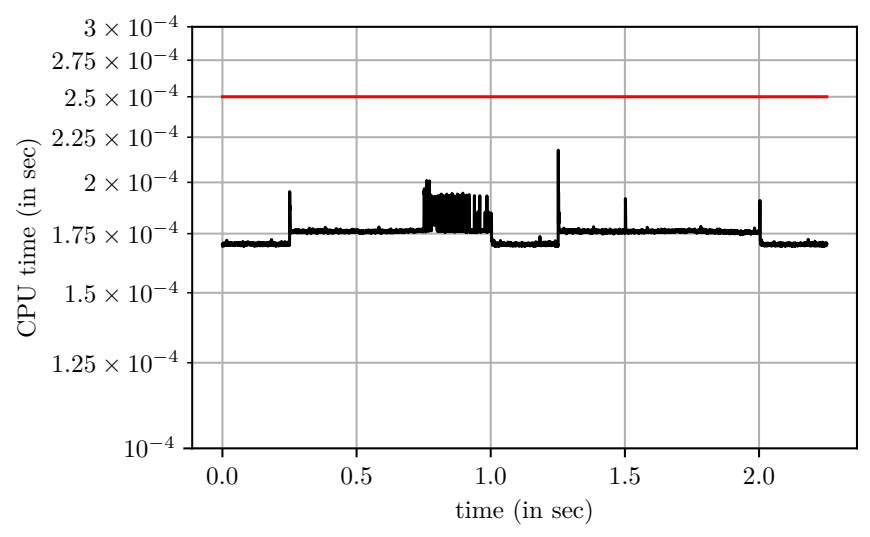

Figure 10: Current steps at $157 \frac{\mathrm{rad}}{\mathrm{s}}$ : overall control loop turnaround time (in black) obtained with the CS-NMPC controller using acados with gPOASES (available computation time of $250 \mu \mathrm{s}$ in red). About $90 \%$ of the computation time is due the CS-NMPC controller (together with the EKF).

a slightly increased reference speed $\omega_{\mathrm{m}, \mathrm{ref}}=165 \frac{\mathrm{rad}}{\mathrm{s}}$.

\section{EXPERIMENTAL RESULTS}

The presented NMPC scheme has been deployed on a custom-built $9.6 \mathrm{~kW}$ RSM (Courtesy of Prof. Maarten Kamper, Stellenbosch University, South Africa) with the parameters reported in Table [1 and the nonlinear flux linkage maps as depicted in Figure 1 (maps were obtained from FEM). The overall laboratory setup is depicted in Figure 3 and comprises the ISPACE real-time system with processor board DS1007 and various extensions and I/O boards, two $22 \mathrm{~kW}$ SEW inverters in back-to-back configuration sharing a common DC-link. Moreover, it comprises the HOSTPC running MATLAB/Simulink with RCPHIL R2017 and dSPACE ControlDesk $6.1 \mathrm{p} 4$ for rapid-prototyping, data acquisition and evaluation, the custom-built $9.6 \mathrm{~kW}$ RSM as device under test and a $14.5 \mathrm{~kW}$ SEW PMSM as load machine. The DR2212 torque sensor allows to measure the mechanical torque too, but it was not used during the experiments. The CS-NMPC controller based on the formulation described in Section III and implemented using the acados framework has been deployed on the ISPACE unit connected to the physical RSM.

In order to validate the control performance of the proposed CS-NMPC controller we reproduced the scenario used for the simulation reported in Section III] i.e., we used the PMSM to maintain the nominal rotational speed of the rotor $\left(157 \frac{\mathrm{rad}}{\mathrm{s}}\right)$ and different torque references have been fed to the RSM controllers under analysis.

The closed-loop trajectories for the conducted experiments are reported in Figure 8.9. Similarly to the results obtained in simulation (see Figures 6 and 77, the proposed CS-NMPC controller achieves better tracking performance than the gainscheduled PI controller, in particular when the voltage constraint becomes active (especially between $t=0.75 \mathrm{~s}$ and $t=1.00 \mathrm{~s}$ ). Notice that there is a non negligible discrepancy between simulation and experimental results potentially due to model mismatches. Among other possible causes of discrepancies, we mention the fact that the modelled flux maps

\begin{tabular}{llll}
\hline par. & value & par. & value \\
\hline$R_{\mathrm{s}}$ & $0.4 \Omega$ & $\omega_{\mathrm{m}, \text { nom }}$ & $157.07 \frac{\mathrm{rad}}{\mathrm{s}}$ \\
$m_{\mathrm{m}, \text { nom }}$ & $61 \mathrm{Nm}$ & $\hat{\imath}_{\mathrm{s}, \max }$ & $29.7 \mathrm{~A}$ \\
$\hat{u}_{\mathrm{s}, \max }$ & $556 \mathrm{~V}$ & - &
\end{tabular}

Table I: Parameters of physical setup (used in simulation too).

might differ from the real ones. Moreover, we observe from Figure 9 that the DC-link voltage fluctuates around its nominal value. In fact, in the presence of a sudden change in the torque reference, the voltage of the DC-link capacitor can drop if the recharging rate is slower than the discharging rate (behavior not modelled in simulation). Although this behavior is not accounted for in the dynamics of the system used to design the controllers under analysis, in both controllers we exploit the measured DC-link voltage in order to adjust the feasible set. Notice that the $u_{\text {ref }}$ computed by the CS-NMPC controller becomes sometimes infeasible. This infeasibility is consistent with the fact that the quadratic constraint can be violated during transient and will only be satisfied at the steady. However, due to the safety polytopic constraints introduced in (14), we can guarantee that the computed solution will not violate the outer voltage hexagon. At the same time, whenever a voltage reference that lies outside of the circular feasible set is fed to the modulator, a projection onto the disk of radius $\frac{u_{\mathrm{dc}}}{\sqrt{3}}$ is carried out.

\section{CONCLUSIONS}

In this paper we present simulation and experimental results obtained with a CS-NMPC torque controller for RSMs. As opposed to most successful implementations present in the literature, that use instead FS-MPC/NMPC, we show the effectiveness and real-time feasibility of the continuous control set approach. In particular, we show that, using the software implementation of the real-time iteration method for NMPC available in the software package acados, it is possible to deploy the proposed controller on embedded hardware and to meet the challenging sampling times typically required to control electrical drives. We discuss implementation details and report on simulation as well as experimental results which show that the proposed approach can largely outperform stateof-art control methods especially when the input constraints become active.

Future research will involve the investigation of novel numerical methods, e.g., the real-time first-order methods proposed in [57], to speed up the computation times, which are currently still rather long and neither allow for extensions of the optimal control formulations (e.g longer horizons, state or input spaces of higher dimension, etc) nor for deployment on hardware with lower computational power.

\section{REFERENCES}

[1] T. Albin, D. Ritter, N. Liberda, R. Quirynen, and M. Diehl. In-vehicle realization of nonlinear MPC for gasoline two-stage turbocharging airpath control. IEEE Transactions on Control Systems Technology, pages 113, 2017. 
[2] Thomas J. Besselmann, Sture Van de moortel, Stefan Almér, Pieder Jörg, and Hans Joachim Ferreau. Model predictive control in the multi-megawatt range. IEEE Transactions on Industrial Electronics, 63(7):4641-4648, 2015.

[3] R.E. Betz, R. Lagerquist, M. Jovanovic, T.J.E. Miller, and R.H. Middleton. Control of synchronous reluctance machines. IEEE Transactions on Industry Applications, 29(6):1110-1122, 1993.

[4] I Boldea, ZX Fu, and SA Nasar. Torque vector control (TVC) of axially-laminated anisotropic (ALA) rotor reluctance synchronous motors. Electric Machines and Power Systems, 19(3):381-398, 1991.

[5] S. Bolognani, L. Peretti, and M. Zigliotto. Online MTPA control strategy for DTC synchronous-reluctancemotor drives. IEEE Transactions on Power Electronics, 26(1):20-28, 2011.

[6] Abdesselam Chikhi, Mohamed Djarallah, and Khaled Chikhi. A comparative study of field-oriented control and direct-torque control of induction motors using an adaptive flux observer. Serbian Journal of Electrical Engineering, 7(1):41-55, 2010.

[7] G. Cimini, D. Bernardini, S. Levijoki, and A. Bemporad. Embedded model predictive control with certified real-time optimization for synchronous motors. IEEE Transactions on Control Systems Technology, pages 18, 2020.

[8] P. Cortes, M.P. Kazmierkowski, R.M. Kennel, D.E. Quevedo, and J. Rodriguez. Predictive control in power electronics and drives. IEEE Transactions on Industrial Electronics, 55(12):4312-4324, 2008.

[9] M. Diehl. Real-Time Optimization for Large Scale Nonlinear Processes. PhD thesis, University of Heidelberg, 2001.

[10] M. Diehl, H.G. Bock, and J.P. Schlöder. Newtontype methods for the approximate solution of nonlinear programming problems in real-time. In G. Di Pillo and A. Murli, editors, High Performance Algorithms and Software for Nonlinear Optimization, pages 177-200. Kluwer Academic Publishers B.V., 2002.

[11] M. Diehl, R. Findeisen, and F. Allgöwer. A stabilizing real-time implementation of nonlinear model predictive control. In L. Biegler, O. Ghattas, M. Heinkenschloss, D. Keyes, and B. van Bloemen Waanders, editors, RealTime and Online PDE-Constrained Optimization, pages 23-52. SIAM, 2007.

[12] M. Diehl, R. Findeisen, F. Allgöwer, H. G. Bock, and J. P. Schlöder. Nominal stability of the real-time iteration scheme for nonlinear model predictive control. IEE Proc.-Control Theory Appl., 152(3):296-308, 2005.

[13] M. Diehl, D.B. Leineweber, and A.A.S. Schäfer. MUSCOD-II Users' Manual. IWR-Preprint 2001-25, University of Heidelberg, 2001.

[14] A. Domahidi, S. Mariethoz, and M. Morari. Highbandwidth explicit model predictive control of electrical drives. IEEE Transactions on Industry Applications, 48(6):1980-1992, November 2012.

[15] Hisham Eldeeb, Christoph M. Hackl, Lorenz Horlbeck, and Julian Kullick. A unified theory for optimal feedforward torque control of anisotropic synchronous machines. International Journal of Control, 91(10):22732302, 2017.

[16] T. Englert and K. Graichen. A fixed-point iteration scheme for model predictive torque control of PMSMs. In Proceedings of the 6th IFAC Conference on Nonlinear Model Predictive Control, volume 51, pages 568-573, Madison, Wisconsin, USA, August 2018.

[17] C. Feller and C. Ebenbauer. Relaxed logarithmic barrier function based model predictive control of linear systems. IEEE Transactions on Automatic Control, 62(3):1223-1238, March 2017.

[18] H. J. Ferreau, H. G. Bock, and M. Diehl. An online active set strategy to overcome the limitations of explicit MPC. International Journal of Robust and Nonlinear Control, 18(8):816-830, 2008.

[19] H. J. Ferreau, C. Kirches, A. Potschka, H. G. Bock, and M. Diehl. qpOASES: a parametric active-set algorithm for quadratic programming. Mathematical Programming Computation, 6(4):327-363, 2014.

[20] Jonathan Frey, Rien Quirynen, Dimitris Kouzoupis, Gianluca Frison, Jens Geisler, Axel Schild, and Moritz Diehl. Detecting and exploiting Generalized Nonlinear Static Feedback structures in DAE systems for MPC. In Proceedings of the European Control Conference (ECC), 2019.

[21] G. Frison, D. Kouzoupis, T. Sartor, A. Zanelli, and M. Diehl. BLASFEO: Basic linear algebra subroutines for embedded optimization. ACM Transactions on Mathematical Software (TOMS), 44(4):42:1-42:30, 2018.

[22] Gianluca Frison and Moritz Diehl. HPIPM: a highperformance quadratic programming framework for model predictive control. In Proceedings of the IFAC World Congress, Berlin, Germany, July 2020.

[23] Tobias Geyer. Model Predictive Control of High Power Converters and Industrial Drives. John Wiley \& Sons, 2016.

[24] Tobias Geyer, Georgios Papafotiou, and Manfred Morari. Model predictive direct torque control - Part I: Concept, algorithm, and analysis. IEEE Transaction on Industrial Electronics, 56(6):1894-1905, 2009.

[25] K. Graichen and A. Kugi. Stability and incremental improvement of suboptimal MPC without terminal constraints. IEEE Transactions on Automatic Control, 55(11):2576-2580, 2010.

[26] Lars Grüne and Dragan Nesić. Optimization-based stabilization of sampled-data nonlinear systems via their approximate discrete-time models. SIAM J. Control Optim, 42(1):98-122, 2003.

[27] Christoph M. Hackl. Current PI-funnel control with antiwindup for synchronous machines. In Proceedings of the 54th IEEE Conference on Decision and Control, pages 1997-2004, 2015.

[28] Christoph M. Hackl. Non-identifier Based Adaptive Control in Mechatronics. Springer International Publishing, 2017.

[29] Christoph M. Hackl, Maarten J. Kamper, Julian 
Kullick, and Joshua Mitchell. Nonlinear PI current control of reluctance synchronous machines. arxiv.org/pdf/1512.09301.v1, 2015.

[30] C.M. Hackl, M.J. Kamper, J. Kullick, and J. Mitchell. Current control of reluctance synchronous machines with online adjustment of the controller parameters. In 2016 IEEE 25th International Symposium on Industrial Electronics (ISIE). IEEE, jun 2016.

[31] B. Houska, H. J. Ferreau, and M. Diehl. ACADO toolkit - an open source framework for automatic control and dynamic optimization. Optimal Control Applications and Methods, 32(3):298-312, 2011.

[32] Marten J. Kamper, F.S. van der Merwe, and S. Williamson. Direct finite element design optimisation of the cageless reluctance synchronous machine. IEEE Transactions on Power Conversion, 11(3):547-555, 1996.

[33] D. Kouzoupis, G. Frison, A. Zanelli, and M. Diehl. Recent advances in quadratic programming algorithms for nonlinear model predictive control. Vietnam Journal of Mathematics, 46(4):863-882, 2018.

[34] R. Lagerquist, I. Boldea, and T.J.E. Miller. Sensorlesscontrol of the synchronous reluctance motor. IEEE Transactions on Industry Applications, 30(3):673-682, 1994.

[35] Peter Landsmann, Ralph Kennel, Hugo W. de Kock, and Marten J. Kamper. Fundamental saliency based encoderless control for reluctance synchronous machines. In Proceedings of the XIX International Conference on Electrical Machines (ICEM), pages 1-7, 2010.

[36] Peter Landsmann, Dirk Paulus, Peter Stolze, and Ralph Kennel. Reducing the parameter dependency of encoderless predictive torque control for reluctance machines. In Proceedings of the IEEE International Symposium on Sensorless Control for Electrical Drives (SLED), pages 93-99, 2010.

[37] T. Matsuo and T.A. Lipo. Field oriented control of synchronous reluctance machine. In Power Electronics Specialists Conference, 1993. PESC '93 Record., 24th Annual IEEE, pages 425-431, Jun 1993.

[38] Manfred Morari and Urban Maeder. Nonlinear offset-free model predictive control. Automatica, 48(9):2059-2067, 2012.

[39] K. R. Muske and T. A. Badgwell. Disturbance modeling for offset-free linear model predictive control. Journal of Process Control, 12:617-632, 2002.

[40] J. Nocedal and S. J. Wright. Numerical Optimization. Springer Series in Operations Research and Financial Engineering. Springer, 2 edition, 2006.

[41] G. Pannocchia, M. Gabiccini, and A. Artoni. Offset-free MPC explained: novelties, subtleties, and applications. In Proceedings of the 5th IFAC Conference on Nonlinear Model Predictive Control, volume 48, pages 342-251, 2015.

[42] G. Pannocchia and J.B. Rawlings. Disturbance Models for Offset-Free Model-Predictive Control. AIChE Journal, 49:426-437, 2003.

[43] D. E. Quevedo, R. Aguilera, and T. Geyer. Model predictive control for power electronics applications. In S. V. Rakovic and W. S. Levine, editors, Handbook of Model Predictive Control, pages 551-580. Birkhäuser, Cham, 2019.

[44] R. Quirynen, S. Gros, and M. Diehl. Efficient NMPC for nonlinear models with linear subsystems. In Proceedings of the IEEE Conference on Decision and Control (CDC), pages 5101-5106, 2013.

[45] R. Quirynen, S. Gros, and M. Diehl. Lifted implicit integrators for direct optimal control. In Proceedings of the IEEE Conference on Decision and Control (CDC), pages 3212-3217, 2015.

[46] R. Quirynen, S. Gros, and M. Diehl. Inexact Newton-type optimization with iterated sensitivities. SIAM Journal on Optimization, 28(1):74-95, 2018.

[47] R. Quirynen, M. Vukov, and M. Diehl. Auto generation of implicit integrators for embedded NMPC with microsecond sampling times. In Mircea Lazar and Frank Allgöwer, editors, Proceedings of the 4th IFAC Nonlinear Model Predictive Control Conference, pages 175-180, 2012.

[48] E.M. Rashad, T.S. Radwan, and M.A Rahman. A maximum torque per ampere vector control strategy for synchronous reluctance motors considering saturation and iron losses. In Conference Record of the 2004 IEEE Industry Applications Conference (39th IAS Annual Meeting), volume 4, pages 2411-2417, 2004.

[49] J. B. Rawlings, D. Q. Mayne, and M. M. Diehl. Model Predictive Control: Theory, Computation, and Design. Nob Hill, 2nd edition edition, 2017.

[50] Bartolomeo Stellato, Goran Banjac, Paul Goulart, Alberto Bemporad, and Stephen Boyd. OSQP: An operator splitting solver for quadratic programs. Mathematical Programming Computation, 2020.

[51] Robin Verschueren, Gianluca Frison, Dimitris Kouzoupis, Niels van Duijkeren, Andrea Zanelli, Rien Quirynen, and Moritz Diehl. Towards a modular software package for embedded optimization. In Proceedings of the IFAC Conference on Nonlinear Model Predictive Control (NMPC), 2018.

[52] Robin Verschueren, Niels van Duijkeren, Rien Quirynen, and Moritz Diehl. Exploiting convexity in direct optimal control: a sequential convex quadratic programming method. In Proceedings of the IEEE Conference on Decision and Control (CDC), 2016.

[53] Longya Xu, Xingyi Xu, Thomas A. Lipo, and Donald W. Novotny. Vector control of a synchronous reluctance motor including saturation and iron losses. IEEE Transactions on Industrial Applications, 27(5):977-985, 1991.

[54] S. Yamamoto, J.B. Adawey, and T. Ara. Maximum efficiency drives of synchronous reluctance motors by a novel loss minimization controller considering crossmagnetic saturation. In Proceedings of the 2009 IEEE Energy Conversion Congress and Exposition, pages 288 293, 2009.

[55] A. Zanelli, G. Horn, G. Frison, and Moritz Diehl. Nonlinear model predictive control of a human-sized quadrotor. In Proceedings of the European Control Conference 
(ECC), pages 1542-1547, 2018.

[56] A. Zanelli, R. Quirynen, and M. Diehl. Efficient zeroorder NMPC with feasibility and stability guarantees. In Proceedings of the European Control Conference (ECC), Naples, Italy, June 2019.

[57] A. Zanelli, Q. Tran-Dinh, and M. Diehl. Contraction estimates for abstract real-time algorithms for NMPC. In Proceedings of the IEEE Conference on Decision and Control (CDC), Nice, France, Dec 2019.

[58] A. Zanelli, Q. Tran-Dinh, and M. Diehl. A Lyapunov function for the combined system-optimizer dynamics in nonlinear model predictive control. arXiv preprint, 2020. (submitted).

[59] A. Zanelli, Q. Tran-Dinh, and M. Diehl. Stability analysis of real-time methods for equality constrained NMPC. In Proceedings of the IFAC World Congress, Berlin, Germany, July 2020.

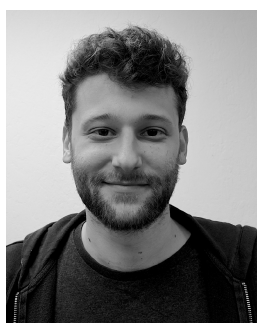

Andrea Zanelli received the B.Sc degree in Automation Engineering from Politecnico di Milano and the M.Sc in Robotics, Systems and Control from ETH Zurich in 2012 and 2015, respectively. Since 2015, he is a Ph.D student at the System, Control and Optimization Laboratory at the University of Freiburg, Germany. His research work focuses on the development and software implementation of efficient numerical methods for embedded optimization and nonlinear model predictive control with numerical and system theoretic guarantees.

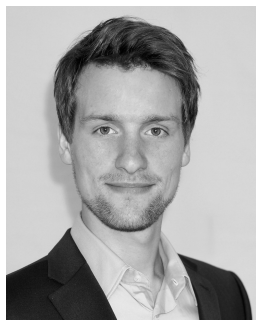

Julian Kullick received the B.Sc. and M.Sc. degrees in electrical engineering from Technical University of Munich (TUM), Munich, Germany in 2012 and 2015 respectively. He is currently working toward the Ph.D. degree in electrical engineering with TUM. From 2015 to 2018, in the course of the "Geothermal-Alliance Bavaria (GAB)" project, he was a Research Associate with the research group "Control of Renewable Energy Systems (CRES)" at TUM. Since 2019 he is a Research Associate with the "Laboratory for Mechatronic and Renewable Energy Systems (LMRES)", Munich University of Applied Sciences, Munich, Germany. His research interests include nonlinear modeling, efficient operation and sensorless control of electric drives.

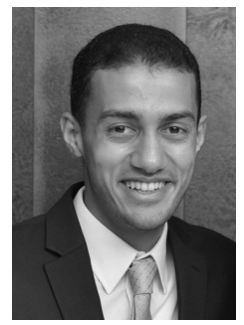

Hisham M. Eldeeb received his B.Sc. (honors) and M.Sc. in electrical engineering in 2011 and 2014, respectively, from the Faculty of Engineering, Alexandria University, Egypt. From 2012 to 2015, he worked as a research associate at Qatar University in Qatar; aiming at extending the penetration of inverter-based distributed-generation plants in the Qatari-Network. From September 2015, he was selected as one of the 14 Marie-Curie Ph.D. candidates, hired on the Horizon H2020 project "Airborne Wind Energy System Control and Optimization" (AWESCO), and earned his Ph.D degree at the Technical University of Munich (TUM) in Germany. His Ph.D topic was related to multi-phase drives and the development of fault-tolerant control strategies. He is currently working as an electrical drive specialist and development engineer for e-vehicles at IAV $\mathrm{GmbH}$. His research interests are grid-connected converters, power electronics, and multi-phase drives.

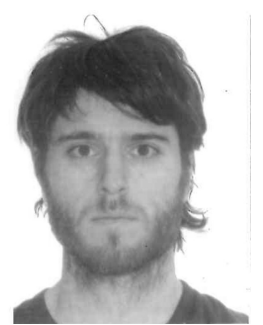

Gianluca Frison received the B.Sc. in Information Engineering in 2009 and the M.Sc. in Automation Engineering in 2012, both from University of Padua. In 2012 he also received the M.Sc. in Mathematical Modeling and Computation, followed in 2016 by the Ph.D, both from Technical University of Denmark. Since January 2016 he has been Post-Doc, alternatively at University of Freiburg, at Technical University of Denmark, and again at University of Freiburg. His current research interests include highperformance linear algebra for embedded optimization and fast numerical methods for model predictive control. He is the main developer of the software packages BLASFEO and HPMPC/HP IPM.

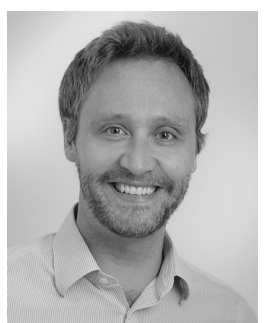

Christoph M. Hackl (M'12-SM'16) studied Electrical Engineering (with focus on controls and mechatronics) at Technical University of Munich (TUM), Germany and University of Wisconsin-Madison, USA, and received the B.Sc., Dipl.-Ing., and Dr.Ing. (Ph.D.) degrees in Electrical Engineering in 2003, 2004 and 2012, respectively, from TUM. Since 2004, he has been teaching electrical drives, power electronics, and mechatronic \& renewable energy systems. Since 2014, he has been the head of the research group "Control of Renewable Energy Systems (CRES)" at TUM. In 2018, he became a Professor for Electrical Machines and Drives and the head of the "Laboratory for Mechatronic and Renewable Energy Systems (LMRES)" at the Munich University of Applied Sciences (MUAS), Germany. His research interests include nonlinear, adaptive and optimal control of electrical, mechatronic and renewable energy systems.

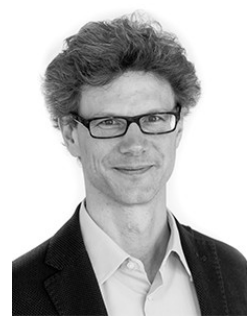

Moritz Diehl graduated with a Diploma in physics and mathematics at Heidelberg University, Heidelberg, Germany and Cambridge University, Cambridge, U.K. in 1999, and received his Ph.D. degree from Heidelberg University in 2001. From 2006 to 2013, he was a Professor with the Department of Electrical Engineering, KU Leuven, Leuven, Belgium. In 2013, he moved to the University of Freiburg, Freiburg, Germany, where he is currently the Head of the Systems Control and Optimization Laboratory, Department of Microsystems Engineering, and the Department of Mathematics

\section{APPENDIX}

\section{ADDITIONAL SIMULATION AND EXPERIMENTAL RESULTS}

In the following, we report additional simulation and experimental (for CS-NMPC only) results carried out at a reference speed of $165 \frac{\mathrm{rad}}{\mathrm{s}}$. In this scenario, the impact of the input constraint is even stronger than for the one used in Sections IIII and IV. Since the results showed potentially damaging behavior when controlling the RSM with the gain-scheduled PI, we ran the corresponding experiments only with CSNMPC. The simulation and experimental results are reported in Figures 11, 12 and 13 These additional results confirm the observation made for the scenario reported in Sections III and IV] 

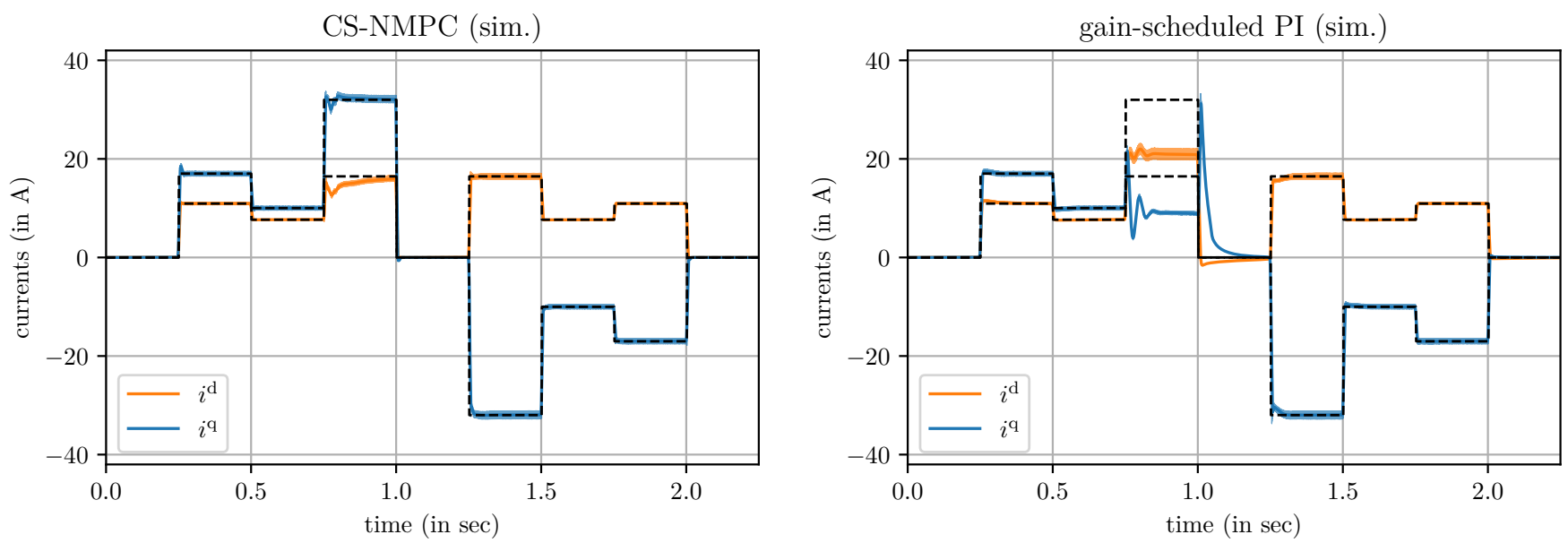

Figure 11: Current steps at $165 \frac{\mathrm{rad}}{\mathrm{s}}$ (simulation): closed-loop trajectories obtained using CS-NMPC (left) and gain-scheduled PI controller (right).
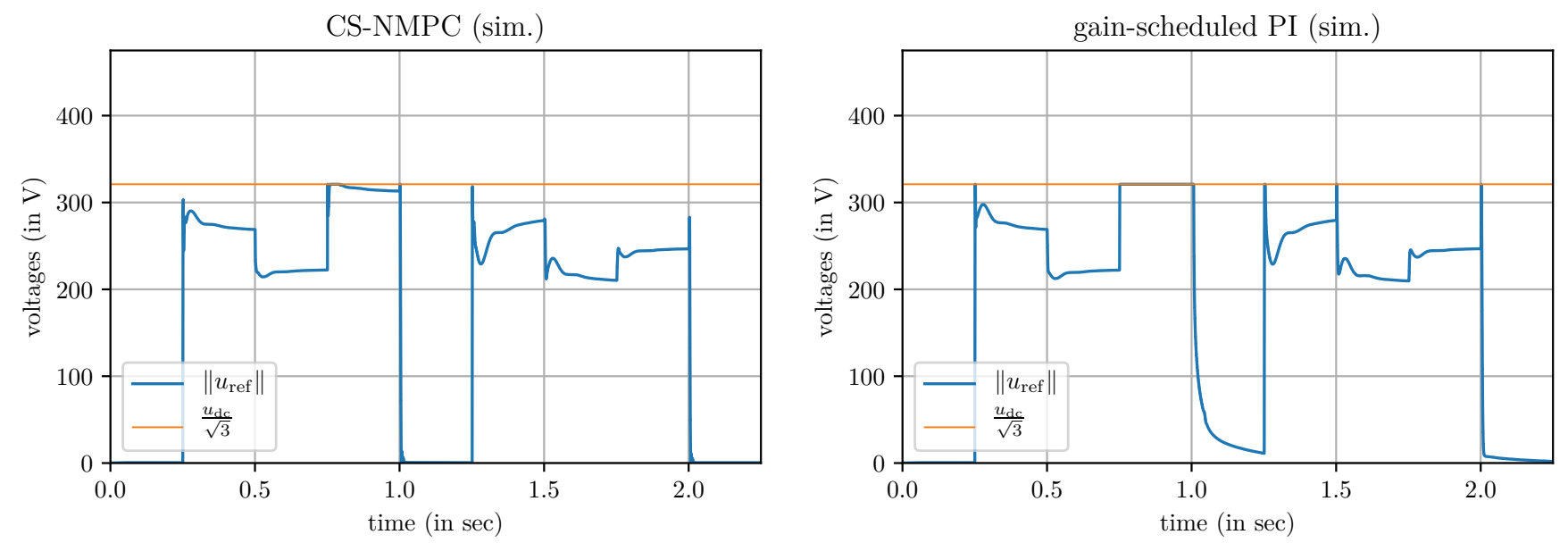

Figure 12: Current steps at $165 \frac{\mathrm{rad}}{\mathrm{s}}$ (simulation): two-norm of voltage references $u_{\text {ref }}$ commanded by the two controllers and $u_{\mathrm{dc}}$ over time. During the third current step, the PI controller saturates and does not steer the system to the desired reference.
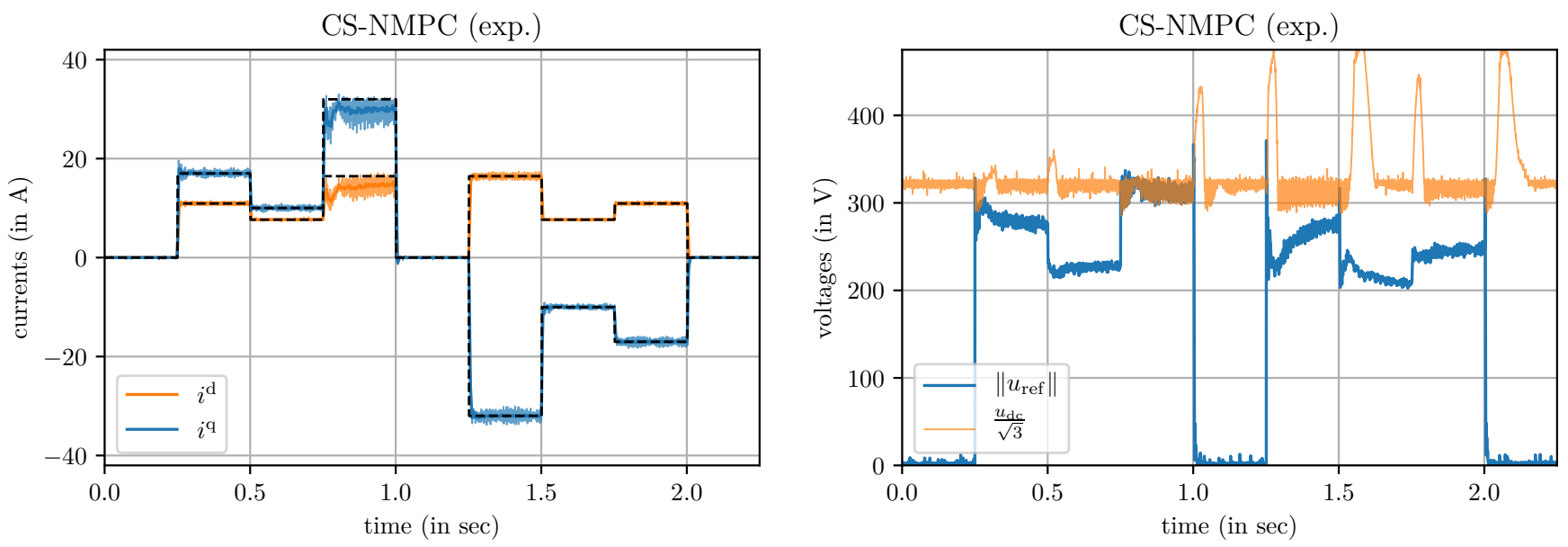

Figure 13: Current steps at $165 \frac{\mathrm{rad}}{\mathrm{s}}$ (experiment): closed-loop current trajectories obtained using the two controllers under analysis. Due to the strong effect of input saturation observed in simulation, for this value of the reference speed it was not possible to run the experiment with the PI controller. 